\title{
REALISM IN CONTEXT: THE EXAMPLES OF LIFEWORLD AND QUANTUM PHYSICS
}

\author{
GREGOR SCHIEMANN
}

\begin{abstract}
Lifeworld realism and quantum-physical realism are taken as experience-dependent conceptions of the world that become objects of explicit reflection when confronted with context-external discourses. After a brief sketch of the two contexts of experience-lifeworld and quantum physics-and their realist interpretations, I will discuss the quantum world from the perspective of lifeworld realism. From this perspective, the quantum world-roughly speaking-has to be either unreal or else constitute a different reality. Then, I invert the perspective and examine the lifeworld from the standpoint of quantumphysical realism. This conception of the lifeworld has gained momentum from new research results in recent decades. Despite its experiential basis, quantum-physical realism bears an ambiguity akin to that of lifeworld realism. While the perspectival inversion serves to highlight the problem, it also contributes to an improved understanding of lifeworld-realism.
\end{abstract}

Keywords: lifeworld; quantum physics; philosophy of science; realism.

\section{Introduction}

One of the most intensively debated topics in epistemology in recent years has been the issue of realism. Discussions have been highly controversial and have been carried out with a marked domain-specificity. The term "realism" refers to the view that objects and properties exist independently of our mental access to them. The ways in which mental processes relate to the objects and properties in the world, however, are quite diverse, as is reflected by the plurality of discourses. From one domain to the next, there are different realism-discourses, which to some extent overlap but are also in some respects mutually exclusive. It has become, for example, common to distinguish among discourses about the reality of macroscopic objects in time and space, about the truth of scientific theories and the reality of their objects, about the reality of mathematical objects, about the reality of moral values and about the reality of the objects of religious attitudes. ${ }^{1}$

This differentiation has shed light upon the object dependency of realist and anti-realist positions, and thereby prepared the way for a step that, although it has yet to be taken, I consider indispensable to clarifying the issue of realism. What I have in mind is a comparative investigation of the arguments specific to different domains. Comparative analyses are necessary insofar as they promise to highlight the relations obtaining among the different discourses and thereby help to make the realism debate do justice to the phenomena.

1 For an overview of the various realism-discourses, see Miller (2008) or Willaschek (2000). 
But a comparative investigation is no mere supplement to existing discourses; rather, it also opens up a new perspective upon them. My thesis is that external discourse-relations are among the conditions responsible for making realism into a problem within specific domains. To put it in somewhat stronger terms, albeit with restricted applicability: on the one hand, reality only becomes a problem when controversies arise among different modes of experience; on the other hand, realism-discourses that integrate external contextual aspects take on a key role in solving the specific problems relating to realism. As little as realism is discussed in connection with external discourse-relations, it cannot be adequately illuminated without reference to them.

In order to pursue this conjecture, I am going to be discussing the examples of realist views concerning the lifeworld, on the one hand, and quantum physics on the other. The lifeworld and quantum physics are an unlike pair. Current lifeworld structures bear similarities with everyday experiences that go back as far as antiquity, whereas quantum physics has only been around for a bit more than 100 years. In this short time-span, it has had a leading role in the development of an astonishing array of technical innovations that cut deep into the world of everyday experience. Dealing with these innovations in everyday life, however, does not require one to have any understanding of how they function. Just about everyone is familiar with experience of the lifeworld, which tends not to be the subject of explicit reflection (outside of philosophy), whereas quantum physics has remained mysterious even to readers of the many high-circulation books that have been written about it.

I choose these two modes of experience because they present two extremes along a spectrum of contexts available to a comparative approach: while lifeworld realism refers to the objects of perception, to what is immediately present to the intuition of the senses, quantum-physical realism refers to the smallest entities in the realm of the unperceivable. This difference corresponds to the distance between the theories that typically address the two modes of experience in philosophy: the structures of our experience of the lifeworld are the preferred topic of phenomenology, whereas the structures of experience accessible by experimentation in quantum physics are predominately the province of analytically oriented philosophy of science. These two modes of experience are also well-suited to a comparative analysis for another reason: the relations between the two contexts play a significant role among the external sources of the problems relating to realism in both specific contexts.

Of course, even despite the restriction to two domains of experience, the matter remains so complex that I can only present it schematically. In order to grasp the lifeworld and quantum physics in a conceptually uniform manner, I will assume a broad concept of experience: it comprehends the processes of knowledge acquisition and the forms of established possession of ordered contents. On the other hand, I will attempt to define the two contexts as narrowly as possible with the help of an ideal-type.

In the first section of the text, I will begin with a brief sketch of the two contexts of experience and the realist understandings prevailing within them. In the second section, I will discuss the quantum world from the perspective of lifeworld realism. This viewpoint presents a historical starting point for the problem of realism with respect to quantum mechanics, which unfolded in the first half the twentieth century against the backdrop of the only gradually changing structures of the lifeworld. From the perspective of lifeworld realism, the quantum world-roughly speaking - has either to be unreal or to constitute a different, only partially conceivable reality.

In the third section, I invert the perspective and examine the lifeworld from the standpoint of quantum-physical realism. This physicalist conception of the lifeworld has gained considerable momentum from new research results in recent decades, and must be taken seriously evenindeed especially — by those who, like myself, are critically disposed to a naturalist outlook. 
Despite its experiential basis, quantum-physical realism bears an ambiguity akin to that of lifeworld realism. While the perspectival inversion serves to highlight the problem, it also contributes to an improved understanding of lifeworld realism.

\section{Two Experiential Contexts and their Realist Understandings}

\section{Lifeworld Realism}

Taking my cue from Edmund Husserl and Alfred Schütz, I conceive of the lifeworld as a world of perception. In the lifeworld, conscious attention is directed toward the practical interaction with familiar things and people as they appear to outer perception. Experience in the lifeworld can be defined via a catalogue of necessary and, taken together, sufficient criteria, such as the orientation of (normal adult) attention to outer perception, a non-professionalized type of action and a holistically structured background knowledge. ${ }^{2}$

The background knowledge encompasses assumptions about what is real that are characteristic of an everyday conception of the world. These assumptions can be divided into an ontological and an epistemological part. Ontologically, an object is regarded as a real entity when existence and intrinsic properties are ascribed to it in itself, i.e. independently of our perception of it. Epistemologically, lifeworld realism stipulates that the properties of an entity can only be known through perception. For the testimony of perception, real objects are localized in time and space and their properties separated from the properties of other objects.

Such a realism, which is related to the conceptions of William James, John McDowell and recently also Hilary Putnam, can be dubbed "direct realism" (James 1912; McDowell 1994; Putnam 1994). The distinction between perception and illusion, on the one hand, is conceived on this view as immanent to the lifeworld, and is based upon conventional standard conditions of perception. On the other hand, the lifeworld is distinct from certain other types of experience, such as children's play, insanity and religious meditation, to which it ascribes only a limited reality. It is essentially in confrontation with other types of experience that direct realism temporarily loses its otherwise unquestionable, self-evident character. From a historical point of view, one can observe that a lasting problematization of direct realism has set in-due to the redemptive doctrines of monotheistic religions, which assert the unreality and wrongheadedness of everyday life, and also to the epistemological criticism that arose with the early modern foundation of the natural sciences, paradigmatically formulated by René Descartes and then developed further by John Locke, David Hume and Immanuel Kant

Before I turn to quantum-physical realism, I would like to take a brief look at one example of this trend, namely at Descartes's critique of lifeworld realism, which had an epochmaking impact on modern natural science's retreat from an everyday understanding of reality. Descartes's critique focuses on the everyday understanding of sensations that accompany perception, and can be illustrated by his reflections on the phenomenon of color. According to Descartes, most people think that when they perceive a color, the external object of perception is similar to the internal sensation of color that simultaneously arises. One takes oneself to know a property of the entity through perception. But this assumption, in Descartes's view, can be "regarded as the first and primary cause of all our errors" (Descartes 1969 ff., VIII, 39). For a precise analysis of our ideas shows that the causes of color-sensation cannot be ascertained from perception. The consciousness of sensations must be conceived as a purely mental phenomenon,

2 For more on the concept of the lifeworld as a world of perception, see Schiemann (2005, $89 \mathrm{ff})$. 
which is not similar to any property or object that it refers to. The material objects of sensation, like all material media in space, are characterized exclusively by extension and motion. Spatiotemporal changes in material suffice to account for all sensations. For Descartes, then, the realistic conception of sensations is to be rejected.

\section{Quantum-physical Realism}

A continuation of the modern critique of lifeworld realism takes its departure from quantum physics, which I would like to characterize as a second experiential context with its own typical realism problem.

By way of a first approximation, one can say that quantum physics is the physics of the very small, which is isolated from its natural surroundings and realized in the displays of measuring devices. From a lifeworld point of view, there is not much to see in the laboratories where micro-physical experiments are conducted other than displays on technical devices, needle deflections and numerical readings. Quantum physics is an experimental study of nature by which knowledge is gained in manipulable technical contexts in order to carry out the minimalization of parameters and variables that is necessary for mathematical treatment. Quantum-physical theory encompasses quantum mechanics and quantum field theory. I will confine myself in the following to quantum mechanics, which is distinct from quantum field theory in that it presupposes that the number of quantum systems is constant.

Classical physics provides the lifeworld with a sort of staging post in the immediate vicinity of the quantum world. For "classical physics" includes the theories that are drawn upon to describe measurement devices, and which are compatible with an everyday understanding of the lifeworld that ascribes intrinsic properties to objects. But the dependence of the physics of the very small upon measurement devices also reveals a fundamental difference between experience in the lifeworld and in quantum physics: quantum objects cannot be perceived but only observed. "Perception" refers here to the perception of external objects as mediated by the senses, whereas "observation" is understood to apply to the empirical reference to theoretical entities as mediated by instruments.

The issue of realism in the natural sciences applies to the truth of theories and to the reality of the entities postulated by theories-i.e. photons, electrons and electromagnetic fields, to name only a few. Analogously to the lifeworld, one can distinguish an ontological from an epistemological realism. Ontologically, the claim would pertain to a reality independent of our knowledge and to the existence of objects that correspond to theoretical entities. Epistemologically, the claim asserts the possibility of knowledge of the properties of these objects and the truth of the theories describing them. The quantum-mechanical problem of realism is linked to lifeworld realism via the question of the role of perception in knowledgeproduction: what epistemological function does perception have in quantum physics?

This question leads me to the second section of my paper, in which I will look at the quantum world from the perspective of lifeworld realism. This perspective, as we shall see, opens up preliminary ways of understanding the quantum world.

\section{Quantum Physics from the Perspective of the Lifeworld}

\section{Epistemological Anti-realism}

It is quite possible to apply the epistemological realism of the lifeworld to quantum physics: one restricts access to reality only to what can be perceived, namely to the measurement results 
that can be read off from instruments. Since this position impugns all knowledge of nonobservable quantum-mechanical entities, it can be characterized as epistemological anti-realism with respect to these objects. According to this anti-realist position, photons, electrons and such are mere illusions, fictions or constructs useful for bringing order into experience. Insofar as it does not wholly deny the existence of a quantum world, it regards this world as unknowable. Historically, this position preceded quantum physics. It was characteristic of positivistic denials of atomism in the late nineteenth and early twentieth centuries, its most famous proponent likely being Ernst Mach. ${ }^{3}$

\section{Epistemological Realism}

The realism problem first becomes urgent when the quantum world advances its own claims to reality in competition to those of the lifeworld, as it were. To this end, knowledge of the real must be extended beyond what is perceivable. One possibility for expanding the epistemic criterion of reality arising from the lifeworld is to ascribe to quantum mechanical objects the values of measured magnitudes - such as location or momentum - as real properties at the time of measurement.

Given this prerequisite, a single experimental setup can yield the insight that the ontology of the lifeworld is not valid for the quantum world. The experiment I have in mind, specifically, is the well-known double-slit experiment, in which the quantum-mechanical objects in questionpictorially speaking - "pass through" either one or two very narrow adjacent openings.

The experiment has been conducted with individual photons, light-rays composed of numerous photons, components of atoms, atoms and molecules. These objects lend themselves to a demonstration of the famous wave-particle duality: in certain experimental constellations, the objects appear to possess the properties of particles, in others the properties of waves. The expressions "particle" and "wave" are derived from classical physics, and correspond to intuitive, lifeworld conceptions. Particles, like bullets, travel along a defined path that ends in a point of contact; waves spread out homogenously in space and interfere with each other like waves in water. The double-slit experiment shows that quantum-mechanical objects have no properties "in themselves", but only with respect to the measurement arrangements with which they are observed. ${ }^{4}$

In an experiment that was first proposed by John Archibald Wheeler in 1978 and has often been repeated since 1984, the experimenter indeed decides only after the object being investigated has entered the measuring apparatus whether it is to be considered a particle or a wave. If one wanted to describe this process in intuitive terms, one would have to assume in the case that a particle is measured that a wave may have been present before but disappeared instantly at all points in space. ${ }^{5}$

Quantum-mechanical systems are characterized by an indeterminacy, which seemingly breaks down suddenly when a measurement is taken. Prior to the measurement, they can be ascribed no unambiguous location. They "are" not either waves or particles but, rather, neither waves nor

\footnotetext{
3 On Mach's realism, see Schiemann (1999).

4 The double-slit experiment and wave-particle duality are still among the standard topics in philosophy of quantum mechanics. For a recent discussion against the background of the realism debate, see Falkenburg (2007).

5 See Shimony (1988) for a presentation of Wheeler's experimental setup.
} 
particles. In the formal language of quantum mechanics, they are presented in "superposition", i.e. as overlapping components of an abstract function, from which the probability of a measurement result - the appearance of a wave or of a particle — can be calculated.

Moreover, in view of the correlations (so-called Einstein-Rosen-Podolsky-correlations) that obtain among quantum-mechanical objects, one cannot ascribe intrinsic properties to them. One of the instances in which this feature appears is in appropriately prepared two-object systems that have a common magnitude of conservation. This is the case, for example, when two photons are emitted from the same source in opposite directions, and their total angular momentum along one spatial axis is zero, since the magnitudes of their angular momentums are equal and are oriented in opposite directions. ${ }^{6}$ The orientations of the two angular momentums are measured in succession, the spatial axis along which the measurement is taken being ascertainable once the photons have left the source.

The first photon measured yields as a result a random distribution for the adjustment of its angular momentum. The second, as one might expect, yields exactly the opposite adjustment (Espagnat 1979). The randomness is indeed characteristic of measurements of specific events in quantum mechanics. However, opinions diverge as to whether chance is an intrinsic property of quantum-mechanical objects or whether it arises only through measurement. ${ }^{7}$ The correlation arises from the fact that the second photon's direction of angular momentum is only established once that of the first photon has been measured (in the opposite direction). As this correlation cannot (as yet?) be traced back to physical interactions, one cannot draw an analogy to any example from the lifeworld. Macroscopic objects have properties independently of each other. When these properties are correlated, localized causes of that correlation can be found. In the case of quantummechanical systems, however, this possibility is ruled out for reasons I cannot go into here.

The realistic interpretation of the values of measured magnitudes presents an example of an epistemological realism in the domain of quantum physics. According to this realist position, what can be known about the quantum world-the existence of which it presupposes-is not fully, but only partially, dependent upon observation.

Some elements of this position correspond to the so-called "Copenhagen interpretation" of quantum mechanics, which comes in different versions and is widely accepted in physics even today, at least wherever philosophical issues concerning realism are discussed. It can be traced back to the work of Niels Bohr, Werner Heisenberg, Max Born and other founders of quantum mechanics in the 1920's. The term "Copenhagen interpretation", however, was introduced only in the mid-fifties, namely by Werner Heisenberg (see Howard 2004). Heisenberg shared Bohr's view that knowledge about quantum objects could be formulated only in the language of a lifeworld ontology containing spatio-temporally localizable and distinct objects. On this view, classical physics is an idealization of everyday experience of the lifeworld and has, like everyday experience, only limited validity. Although classical physics, being the physics of measuring instruments, precedes quantum-physical knowledge, on this interpretation it is nevertheless-paradoxically_not consistently applicable to quantum-physical objects. The unavoidable classical description remains linked to the specific measurement set-up, which

\footnotetext{
${ }^{6}$ The angular momentum corresponds to the impetus with which a macroscopic body rotates like a spinning top around its own axis.

7 The question of chance is an object of dispute between deterministic and indeterministic interpretations of quantum mechanics, each of which comes in different varieties. See, for example, Rae (2004), Bub (1997).
} 
always only captures particular properties of quantum objects. Wave- and particle-properties, on the Copenhagen interpretation, are at the same time mutually exclusive and also complementary properties-hence Bohr's concept of "complementarity". The quantum world is only partially knowable, but our knowledge does suffice to home in on the view that the lifeworld and the quantum world are different realities. ${ }^{8}$

The reception in history and philosophy of science in recent years has increasingly tended toward the view that the Copenhagen interpretation constitutes a continuation of Kant's transcendental foundation of knowledge. In particular, the recourse to the lifeworld is said to bear similarities to Kant's conditions of the possibility of experience, namely in respect to the forms of intuition - time and space — and the category of causality (Chevalley 1994; Faye 2002; Camilleri 2005; Brock 2003). In my view, however, a comparison of the two conceptions reveals more opposites than commonalities. The limited scope of everyday conditions of knowledge, as claimed by the Copenhagen interpretation, results from an empiricist construal of the concepts of space, time and causality, which runs contrary to Kant's claim to universal validity. Moreover, the difference between the reality of the lifeworld and that of the quantum-physical world, emphasized in particular by Heisenberg, is incompatible with the unity of knowledge, the unshakeable foundation of which was Kant's primary concern (Schiemann 2008, $54 \mathrm{ff}$.).

\section{The Quantum View on the Lifeworld}

As I alluded to earlier, recent research results have undermined the ontological and epistemological distinction between macro- and micro-world. Up until just a few decades ago, most scientists believed that quantum properties only manifested themselves in sub-atomic or atomic dimensions, and that the impressive technical utility that is presently being drawn from quantum physics would even in the future be limited to effectuating phenomena without quantum properties. But new experiments have made it increasingly clear that there is no sharp divide between very small and mid-range orders of magnitude. Quantum properties have been found, for example, in molecular aggregates at the nanometer-level and in macroscopic systems at low temperatures; in areas such as message encryption, communication and information processing, novel technical applications of quantum properties are being developed (Shimony 1988; Bouwmeester et al. 2001; Zeilinger 2003).

Inspired by the confirmation of all quantum-mechanical predictions tested so far and by the ever-larger dimensions of objects with quantum properties, realist interpretations laying claim upon universal validity for quantum mechanics have been gaining new momentum. These interpretations hold that quantum-mechanics needs to account not only for the measuring instruments but also for the surroundings, the consciousness of the observers and, ultimately, the entire universe. ${ }^{9}$ But how would we conceive of quantum phenomena that were also present in the lifeworld?

This question leads into the third section of my paper, which inverts the perspective of the preceding section and examines the lifeworld from the external perspective of quantum-physical realism. Here, as we shall see, it is the reality of the lifeworld that either takes on the status of a special world or is disputed altogether.

8 On the Copenhagen interpretation, see Faye (2002) and Beller (1996); on Heisenberg's interpretation of quantum mechanics, see Schiemann (2008).

9 For an introduction, see Rae (2004), Bub (1997). 
Quantum phenomena present in the lifeworld would indeed appear strange. It would be a matter of quantum-mechanical superpositions on the macroscopic level, i.e. the simultaneous occurrence of mutually exclusive properties in one object. Imagine, for example, material objects concerning which one could not know whether they were localized particles or waves spread out in space; or states of organisms that could not unambiguously be determined, such as a cat that could also be a bird, or that were in two places at once, or were simultaneously alive and dead-to take Erwin Schrödinger's famous thought experiment (Joos 2002; Schrödinger 1935). Why is it that we do not perceive such phenomena in the lifeworld, although they might be quantum-mechanically possible?

Answers based on research of so-called "decoherence" have recently gained considerable force and appear to present a promising direction. "Decoherence" is a technical term that refers to a physical process that dissolves quantum-mechanical superpositions. The important idea behind it is to explain the transition from quantum-mechanical indeterminacy to the physical observation of an unambiguous end-state by appealing to the interaction of quantum-mechanical systems with their environment. ${ }^{10}$

A microscopic system can normally only be measured once it has been scrupulously isolated from its surroundings. Not only the measurement of the object, but also any exchange of material and energy between the object and its surroundings, contributes to undermining this isolation. Such interactions are made responsible for the dissolution of quantum-mechanical superpositions.

Hence, a living cat cannot be a part of a quantum-mechanical system, since it cannot be isolated from its surroundings: it has a body temperature, it breathes, etc. On account of its ineliminable interaction with its surroundings, it would be either alive or dead in Schrödinger's thought experiment before the reading were taken. If, however, one were to replace the cat with a bacterium - to take up a suggestion made by Anton Zeilinger $(2003,101)$ - the chances of producing a quantum-mechanical superposition with a living organism would be better insofar as a bacterium can be more effectively quarantined from environmental influences. Why should it not be possible to register overlapping states of a small organism if this can already be done for molecules at the nanometer-level?

The remarkable thing about research on decoherence is that it has been supported not only by calculations but, for several years now, also by empirical investigation. In special experimental setups, the dissolution of quantum-mechanical superpositions can be observed at impressive time-resolutions. This offers a starting point for philosophy of science to hone a more precise account of the specific nature of quantum-physical experience, facilitating for the first time a theoretical presentation of the experimentally variable conditions of artificially produced isolation.

From an epistemological point of view, decoherence is compatible with various positions that provide realist interpretations of quantum mechanics. One thing that these positions have in common is that they invert the relationship between lifeworld and quantum physics set forth by the Copenhagen interpretation: the realism of the lifeworld no longer precedes that of quantum physics but stands in need of explanation against the background of a microphysical ontology. I would like to sketch two of the ever more numerous examples of strategies for conceptualizing this issue epistemologically.

${ }^{10}$ On the role of decoherence in quantum mechanics, see Bacciagaluppi (2003), Blanchard et al. (2000). 


\section{The Lifeworld as a Reality}

The first strategy seeks to explain lifeworld realism as an irreducible phenomenon on the basis of quantum-mechanical realism. The restriction of superpositions to the domain of quantum physics is traced back to the mechanism of decoherence, by means of which microscopic objects lose their quantum properties through even the slightest interactions with their surroundings (Giulini et al. 1999; Zurek 1991; Joos and Zeh 1985).

This explanation would help to support the realism that motivates it if it could be shown to be uniquely valid. For to the extent that an explanation can be shown to be the only possible one, the conditions for establishing its truth improve. Moreover, explanations that are adequate to the phenomena do provide weighty arguments for realistic interpretations of the corresponding theories. But there are still other empirically equivalent theories available for explaining lifeworld realism.

\section{The Lifeworld as an Illusion}

The second strategy, in contrast to the first, impugns the truth of lifeworld realism. Just as quantum phenomena appear illusory from the perspective of the lifeworld, perception in the lifeworld takes on the character of deception from this standpoint. This strategy also typically appeals to recent results from research on decoherence. Theoretically, superpositions only have to dissolve relative to the particular interaction-one can therefore defend the hypothesis that superpositions continue to exist beyond these partial interactions.

Proceeding from this starting point, the second strategy holds that the entire universe can be grasped with quantum mechanics, whereby superpositions enter into the description of the lifeworld. Hence, lifeworld realism must be wrong in epistemological and ontological respects: perception does not provide the foundation for knowledge of what exists; being has a different structure from that assumed in the lifeworld. Hugh Everett's conception provides an example of this strategy, as do the so-called "many-worlds interpretations" based upon Everett's conception-which have enjoyed a great deal of attention in philosophy of physics as well as among physicists in recent years. This growing interest reflects the persuasive force stemming from the corroboration of quantum-mechanical predictions. Nevertheless, the great attention paid to "many-worlds interpretations" is surprising in light of the high price that they pay in terms of intuitive plausibility. ${ }^{11}$

They attempt to do justice to the assumption of macroscopic superpositions by postulating parallel worlds in which the mutually exclusive properties can simultaneously occur. With every quantum-physical measurement that is only statistically calculable, then, as many new worlds arise as different results are theoretically possible. One is always only in the world corresponding to a particular measurement result, and other results are real in other worlds. The problem with this bizarre conception of reality is to account plausibly for the fact that this plethora of realities completely escapes everyday experience in the lifeworld.

Without going into any great detail, I would like to comment briefly on the conditions of validity for this version of quantum-mechanic realism, too. The assumption of universal existence of superpositions forms the most extreme realist interpretation of quantum mechanics and also the most extreme devaluation of lifeworld realism. Its plausibility can be defended,

\footnotetext{
${ }^{11}$ On Everett's interpretation of quantum mechanics and the "many-worlds interpretation" based thereupon, see Barrett (1999); Bub (1997).
} 
independently of whether it can be grasped from the perspective of the lifeworld, with the help of a prominent argument advanced by Hilary Putnam, according to which it is the explanatory and predictive successes of a theory that justify belief that the theory is true. This argument asserts that such success would amount to a miracle if the theory were not at least approximately true. This is, however, not a strict argument, as even the impressive success of quantum mechanics could be traced to its utility rather than its truth.

\section{Conclusion}

In conclusion, I would like to summarize my central theses and offer an overall assessment.

I take both lifeworld realism and quantum-physical realism as experience-dependent conceptions of the world that only become objects of explicit reflection when confronted with context-external discourses.

The multitude of conceptions of the world that I have assumed here springs from an epistemological antirealism. Divergent descriptions of reality are dependent upon our mental access to reality. On the other hand, I have asserted the domain-specificity of experience, which suggests a structure that is independent of our knowledge. Insofar as conceptions of what is real are influenced by the particularities of their specific domains of experience, the boundaries of their validity are also given. The conception of reality emerging from a domain of experience can strike one as foreign, and can elude familiar modes of understanding. The reality of other domains of experience can also appear to be unreal.

I have presented the lifeworld as a world of perception that is distinct from other types of experience-including experience in the natural sciences. With the onset of modernity, the natural scientific conception of the world has rendered the status of lifeworld realism increasingly problematic. As an example, I sketched Descartes's critique of a realistic conception of color sensations. Despite the criticism emerging from the natural sciences, though, the lifeworld conception of reality remains a significant point of reference for the interpretation of scientific theories. The relationship between lifeworld realism and quantummechanical realism attests to this tension.

If one takes only the perspective of the lifeworld, quantum objects, being inaccessible to perception, appear to be unreal. If, however, one is prepared to drop the restriction of access to reality to perception, quantum phenomena take on a reality of their own. This position, which entered into the so-called Copenhagen interpretation of quantum mechanics, assumes that quantum physics and the lifeworld constitute two distinct domains of experience. The scope of validity of lifeworld realism is limited by the quantum world in a way that cannot be grasped within its mode of understanding.

The conception of two opposing worlds has been shaken in recent years by the demonstration of quantum properties above the atomic level. From the perspective of quantum physics, which is no longer separated sharply from the macroscopic level, lifeworld realism stands in need of explanation. The two explanatory strategies I discussed above both stand in contrast to the approach I have adopted, which seeks to compare domain-specific realisms.

The first accepts lifeworld realism and founds its restricted scope upon the new criterion of object isolation. The scope of validity of quantum-mechanical superpositions, on this view, extends only to states of extreme, artificially produced separation of objects from their surroundings - a separation that does not occur in the lifeworld. This new determination of the difference among domains of experience is well-suited to provide a basis for the juxtaposition of various realisms, to which my approach is committed. From its external perspective upon 
the lifeworld, quantum-mechanical realism also offers an explanation for the limited scope of validity of lifeworld realism, which is not intelligible from within the lifeworld.

The second strategy takes its point of departure from the universality of superpositions, which underlie reality. It rejects positions that question this comprehensive reality and deny any knowledge of it. As an example of this strategy, I presented the many-worlds interpretation of quantum mechanics. The unrestricted claim to validity endorsed by this physicalist position is comparable to that of the lifeworld realism that also regards its conception of reality as absolute and denies the reality of quantum objects.

I regard the mutual rejection that marks this relationship between lifeworld realism and quantum-mechanical realism as the expression of an insufficiently enlightened confrontation. The significant attention that has been paid to the many-worlds interpretation is a symptom of the remoteness of physics from the lifeworld, and highlights the refusal of physics to acknowledge the testimony of non-scientific perception that is in contradiction with scientific theories. Contra this inadmissible generalization, I advocate an awareness of the limited scope of validity of particular realist conceptions. This awareness, though, can only be cultivated by confronting various contexts of experience with each other.

Thus, my remarks are intended not only as a contribution to the description of historical and systematic relations among various realisms, but also as a contribution to stimulating intraphilosophic discourse as well as the discourse between philosophy and other disciplines.

\section{References}

Agazzi, E. Realism and Quantum Physics. Amsterdam: Rodopi, 1997.

Bacciagaluppi, G. The Role of Decoherence in Quantum Mechanics. In E. Zalta (Ed.). The Stanford Encyclopedia of Philosophy, 2003. URL $=<$ http://plato.stanford.edu/archives/sum2005/entries/qmdecoherence/s.

Barrett, J. Everett's Relative-State Formulation of Quantum Mechanics. In E. Zalta (Ed.). The Stanford Encyclopedia of Philosophy, 2003. URL $=<$ http://plato.stanford.edu/entries/qm-everett/ $>$.

Barrett, J. The Quantum Mechanics of Minds and Worlds. Oxford: Oxford University Press, 1999.

Beller, M. The Rhetoric of Antirealism and the Copenhagen Spirit. Philosophy of Science 63(2), 183204, 1996.

Blanchard, P. et al. (Eds.). Decoherence: Theoretical, Experimental and Conceptual Problems. Berlin: Springer, 2000.

Boyd, R. Scientific Realism. In E. Zalta (Ed.). Stanford Encyclopedia of Philosophy, 2002. URL = $<\mathrm{http}: / /$ plato.stanford.edu/entries/scientific-realism/>.

Bouwmeester, D., A. Ekert, A. Zeilinger (Eds.). The Physics of Quantum Information: Quantum Cryptography, Quantum Teleportation, Quantum Computation. Berlin/N. Y.: Springer, 2000.

Bouwmeester, D., A. K. Ekert, A. Zeilinger (Eds.). The Physics of Quantum Information : Quantum Cryptography, Quantum Teleportation, Quantum Computation. Berlin/New York: Springer, 2001.

Brock, S. Niels Bohr's Philosophy of Quantum Physics in the Light of Helmholtzian Tradition of Theoretical Physics. Berlin: Logos Verlag, 2003.

Brown, J. Philosophy of Mathematics: An Introduction to the World of Proofs and Pictures. London/ N.Y.: Routledge, 1999.

Bub, J. Interpreting the Quantum World. Cambridge: Cambridge University Press, 1997.

Camilleri, K. Heisenberg and the Transformation of Kantian Philosophy. International Studies in the Philosophy of Science 19, 271-287, 2005.

Chevalley, C. Niels Bohr's Words and the Atlantis of Kantianism. In J. Faye and H. Folse (Eds.). Niels Bohr and Contemporary Philosophy. Berlin: Springer, 1994. 
d'Espagnat, B. The Quantum Theory and Reality. Scientific American 241, 158-181, 1979.

Descartes, R. Euvres. Pub. par C. Adam et P. Tannery. Paris 1969 ff.

Falkenburg, B. Particle Metaphysics: a Critical Account of Subatomic Reality. Berlin: Springer, 2007.

Faye, J. Copenhagen Interpretation of Quantum Mechanics. In E. Zalta (Ed.). Stanford Encyclopedia of Philosophy, 2002. URL $=<$ http://plato.stanford.edu/entries/qm-copenhagen/>.

Fine, A. Is Scientific Realism Compatible with Quantum Physics? In R. Boyd, P. Gasper and J.D. Trout (Eds.). The Philosophy of Science. Cambridge Mass.: MIT-Press, 1991.

Fine, A. The Shaky Game: Einstein, Realism and the Quantum Theory. Chicago: University of Chicago Press, 1986.

Giulini, D. et al. Decoherence and the Appearance of a Classical World in Quantum Theory. Studies in History and Philosophy of Modern Physics 30B(3), 437-441, 1999.

Heisenberg, W. Physics \& Philosophy: the Revolution in Modern Science. New York: Harper \& Row, 1958.

Howard, Don A. Who Invented the "Copenhagen Interpretation"? A Study in Mythology. Philosophy of Science 71(5), 669-682, 2004.

James, W. Essays in Radical Empiricism. New York: Longman Green and Co., 1912.

Joos, E. Dekohärenz und der Übergang von der Quantenphysik zur klassischen Physik. In J. Audretsch (Ed.). Verschränkte Welt. Weinheim: Wiley-VCH, 2002.

Joos, E., Zeh, H. The Emergence of Classical Properties through Interaction with the Environment. Zeitschrift für Physik B 59, 223-243, 1985.

Landsman, N. Between Classical and Quantum. In J. Earman and J. Butterfield (Eds.). Philosophy of Physics, Amsterdam: Elsevier, 2006.

McDowell, J. Mind and World. Cambridge Mass.: MIT-Press, 1994.

Miller, A. Realism. In E. Zalta (Ed.). The Stanford Encyclopedia of Philosophy, 2008. URL $=<$ http:// plato.stanford.edu/entries/realism/>.

Psillos, S. Scientific Realism: How Science Tracks Truth. London: Routledge, 1999.

Putnam, H. The Dewey Lectures. Journal of Philosophy 91, 445-517, 1994.

Rae, A.I.M. Quantum Physics : Illusion or Reality? Cambridge: Cambridge University Press, 2004.

Redhead, M. Incompleteness, Nonlocality and Realism: a Prolegomenon to the Philosophy of Quantum Mechanics. Oxford: Clarendon Press, 1987.

Schiemann, G. Historische Reflexion als Kritik naturwissenschaftlicher Ontologie. Ernst Machs Kritik an der mechanistischen Auffassung des Energieerhaltungssatzes und ihre Aktualität. In J. NidaRümelin (Eds.). Rationality, Realism, Revision. Berlin/N. Y.: de Gruyter, 1999.

Schiemann, G. Naturalismus und Dualismus als naturphilosophisches Problem. Das Verhältnis von Natur und Erfahrung. In K. Köchy and M. Norwig (Eds.). Denken in Kreisläufen Umweltphilosophie zwischen Ethik und Naturphilosophie. Freiburg: Verlag Karl Albert, 2006.

Schiemann, G. Werner Heisenberg. München: C.H.Beck, 2008.

Schrödinger, E. Die gegenwärtige Situation in der Quantenmechanik. Die Naturwissenschaften 23, 844-849, 1935.

Shimony, A. The Reality of the Quantum World. Scientific American 258, 46-53, 1988.

Willaschek, M. Realismus. Paderborn: Schöningh, 2000.

Zeilinger, A. Einsteins Schleier. Die neue Welt der Quantenphysik. München: Goldmann, 2003.

Zurek, W. H. Decoherence and the Transition from Quantum to Classical. Physics Today 44, 36-44, 1991.

Philosophisches Seminar / FB A,

Bergische Universität Wuppertal,

Gaußstr. 20 D-42119 Wuppertal

Germany

E-mail: schiemann@uni-wuppertal.de 